\title{
Gestion du risque infectieux associé aux soins et services au Centre National Hospitalier et Universitaire Hubert Koutoukou Maga de Cotonou (Bénin)
}

\author{
E-M. OUENDO ${ }^{1 *}$, J. SAIZONOU ${ }^{1}$, C. DEGBEY ${ }^{1}$, C. GLELE KAKAI ${ }^{2}$, Y. GLELE $^{1}$ \\ et M. MAKOUTODE ${ }^{1}$
}

${ }^{1}$ Institut Régional de Santé Publique de Ouidah, Université d’Abomey Calavi, Bénin ; BP 384 Ouidah, Bénin.

${ }^{2}$ Ministère de la Santé, Direction Nationale de la Santé Publique, Service de Surveillance Epidémiologique, BP 04 BP 378 Cotonou, Bénin.

*Auteur correspondant, E-mail : eouendo@yahoo.fr; Tél : +22997979721 ; +22995955495

\section{RESUME}

La qualité des soins est une priorité nationale et les infections associées aux soins un défi pour les acteurs hospitaliers. Toute intervention visant la réduction d'un problème passe par un état des lieux. L'objectif de cette étude était d'évaluer la qualité de la gestion du risque infectieux associé aux soins au Centre National Hospitalier et Universitaire de Cotonou en vue de proposer des mesures pour son amélioration. Une étude transversale rétrospective et évaluative sur 193 patients a été réalisée dans cinq (5) services dudit centre. Le choix était raisonné basé sur les services à risque élevé des infections associées aux soins. La fréquence des patients infectés était de 9,84\%. Les sites de localisation observés étaient dans l'ordre décroissant, les tissus mous, le site urinaire, le sang et le site opératoire. Quinze (15) bactéries ont été dénombrées comme étant responsables des infections dont huit (8) multirésistantes aux antibiotiques usuels. Les principaux germes identifiés étaient Klebsiella pneumoniae, Escherichia coli, Staphylococcus aureus, Enterobacter cloacae et Acinetobacter spp. Le risque d'infections associées aux soins reste une préoccupation majeure avec une fréquence importante de bactéries multi résistantes aux antibiotiques usuels.

() 2015 International Formulae Group. All rights reserved.

Mots clés : Infections associées aux soins, fréquence, bactéries multi résistantes, antibiotique, Cotonou.

\section{INTRODUCTION}

La qualité des soins a toujours été une préoccupation des acteurs du système sanitaire. La prévention des infections associées aux soins (IAS) est une exigence pour la qualité des soins que nous offrons aux populations. La sécurité des soins et l'innocuité demandent une application rigoureuse des règles en matière d'hygiène hospitalière. Il existe un lien étroit entre la défaillance de l'hygiène hospitalière et la survenue des infections associées aux soins
(OMS, 2008 ; Raisin, 2002-2004). Le développement de telles infections montre la faiblesse de notre système à garantir la sécurité des soins aux patients. La prévalence de ces infections n'est pas négligeable même dans les pays développés disposant pourtant d'infrastructures, de matériels et d'équipements adéquats (OMS, 2008 ; Fontaine et al., 2001). Différentes approches sont adoptées pour accroître la qualité des prestations. Ces démarches sont menées à travers l'identification et la résolution 
constante des problèmes rencontrés lors de la mise en œuvre des interventions. Les infections nosocomiales sont les infections acquises durant un épisode de soins administrés par un établissement de santé. Elles constituent, de ce fait, un problème de sécurité majeur pour le patient en milieu hospitalier (Raisin, 2002-2004). L'épidémiologie des infections nosocomiales répond aux grands principes des maladies transmissibles (Fontaine et al., 2001). Les interventions sanitaires ne sont pas dénuées de risque. Les études réalisées entre 2005 et 2006 dans les pays développés en Europe et aux Etats-Unis révélaient que la fréquence des infections associées aux soins variait entre 5 et $15 \%$ des patients hospitalisés; elles peuvent affecter 9 à $37 \%$ des patients admis en unités de soins intensifs avec des taux de mortalité bruts de $12 \%$ à $80 \%$ (Vincent, 2003 ; Pittet et al., 2006 ). En France, l'enquête nationale de prévalence réalisée en 2006, rapportait la prévalence des infections associées aux soins à 5,38\% et celle des infectés à 4,97\% (Vincent et al., 2008). En Afrique, précisément au Maroc, cette prévalence était estimée à 6,7\% en 2007 (El Rhazi et al., 2007). Depuis 2006 au Bénin, des efforts sont déployés pour lutter contre les infections associées aux soins et services, mais les données statistiques sur la question ne sont pas toujours tenues. Des études réalisées par Biaou (2011) sur la prévalence des Infections du Site Opératoire (ISO) dans les services de chirurgie viscérale A\&B du CNHU-HKM et Dégbey et al. (2012) sur la qualité du matériel médico-technique utilisé dans les blocs opératoires en vue de la prévention des infections associées aux soins et services au Centre National Hospitalier et Universitaire de Cotonou ont montré respectivement des fréquences des infections nosocomiales de $6,3 \%$ et de $6,17 \%$. Le risque d'épidémies causées par ces germes reste toujours préoccupant avec une fréquence importante de multi-résistance des bactéries aux antibiotiques du fait de l'accumulation des résistances naturelles ou acquises, ne les rendant plus sensibles qu'à un petit nombre d'antibiotiques usuellement actifs en thérapeutique. Cette étude a pour objectif de déterminer la fréquence des infections associées aux soins au CNHUHKM de Cotonou au Bénin. Les résultats visent la sensibilisation des professionnels de la santé dans le cadre du renforcement des activités de l'hygiène hospitalière.

\section{MATERIEL ET METHODES}

L'étude a été réalisée au CNHU-HKM de Cotonou, hôpital de référence national au Bénin. C'est un hôpital de 617 lits qui s'étend sur 10 hectares. L'objectif de cette étude a été d'évaluer la qualité de la gestion du risque infectieux associé aux soins au Centre National Hospitalier et Universitaire de Cotonou en vue de proposer des mesures pour son amélioration. Il s'est agi d'une étude transversale rétrospective et évaluative réalisée au CNHU-HKM de Cotonou. Cette étude a porté sur 193 patients hospitalisés et qui y ont séjourné pendant plus de 48 heures dans le mois de janvier 2012. Le choix des services a été fait sur la base du niveau de risque d'infection. Les services retenus ont été les chirurgies urologiques et viscérales, la clinique universitaire d'accueil des urgences, les unités des grands brûlés et la néonatologie.

Une exploitation des dossiers cliniques des patients a été faite et un entretien a été réalisé avec les surveillants des services concernés pour recueillir des compléments d'informations. Le registre du laboratoire de microbiologie et les duplicata des résultats de cultures et d'antibiogramme ont été aussi exploités.

Les variables sont regroupées en deux catégories :

-la variable dépendante qui est la qualité de la gestion du risque infectieux ;

-les variables indépendantes que sont:

- la fonctionnalité des structures de promotion d'hygiène hospitalière ;

- la mise en œuvre des barrières en hygiène hospitalière ; 
- la disponibilité des outils de gestion de la prévention des IAS ;

- la Surveillance des Infections Associées aux Soins (IAS) .

\section{Diagnostic des infections associées aux soins et services}

Le diagnostic des infections nosocomiales a été réalisé à travers les informations contenues dans les dossiers cliniques des patients. Il était basé sur les critères internationaux de la définition des infections associées aux soins contenus dans le document des 100 recommandations proposées par le Comité technique national des infections nosocomiales en France et de leur actualisation (CTINILS, 2007).
Les définitions de cas et des localisations des infections étaient basées sur les critères simplifiés de l'OMS pour la surveillance des cas et de l'Agence Nationale d'Accréditation et d'Evaluation en Santé (ANAES) concernant les infections du nouveau-né (OMS, 2008 ; ANAES, 2003). Ainsi, pour apprécier la surveillance des infections associées aux soins et la qualité de gestion du risque infectieux, nous avons retenu les cotations tel que décrit par le Tableau 1 et la synthèse des cotations par variable indépendante (Tableau 2).

Tableau 1 : Les cotations retenues pour apprécier la surveillance des IAS et la qualité de gestion du risque infectieux.

\begin{tabular}{lc}
\hline Modalités & Cotations \\
\hline Signalement des IAS & $\mathbf{4}$ \\
Procédure de signalement des IAS écrite & 1 \\
Diffusion de la procédure de signalement des IAS & 1 \\
Supports de collecte des IAS disponibles & 1 \\
Information/Formation du personnel sur procédure signalement des IAS & 1 \\
\hline Gestion des phénomènes épidémiques ou inhabituels & $\mathbf{3}$ \\
\hline Existence d'un programme (Service ou division) de surveillance épidémiologique des IAS & 1 \\
\hline Fonctionnement du programme de surveillance (Existence du protocole de surveillance : & 1 \\
Enregistrement, Compilation, Analyse); Document faisant état du bilan de la surveillance) & \\
\hline Procédure d'intervention en cas d'épidémie ou de phénomènes inhabituels (Bacilles Multi & 1 \\
\hline \multicolumn{1}{c}{ Résistants_BMR) } & \\
\hline
\end{tabular}

Tableau 2 : La synthèse des cotations par variable indépendante.

\begin{tabular}{lc}
\hline Qualité de gestion du risque infectieux associé aux soins & Cotation attendue \\
\hline $\begin{array}{l}\text { Fonctionnalité des structures de promotion d'hygiène } \\
\text { hospitalière }\end{array}$ & 22 \\
\hline Mise en œuvre des barrières en hygiène hospitalière & 42 \\
\hline $\begin{array}{l}\text { Disponibilité des outils de gestion de la prévention des } \\
\text { Infections associées aux soins }\end{array}$ & 29 \\
\hline Surveillance des Infections associées aux soins & 7 \\
\hline Cotation totale attendue & $\mathbf{1 0 0}$ \\
\hline
\end{tabular}




\section{Surveillance des infections associées aux soins}

\section{Traitement et analyse des données}

Les données recueillies avaient été saisies et analysées par le logiciel Epi info 3.5.3. Le test de $\mathrm{Chi}^{2}$ de Pearson a été utilisé pour tester l'indépendance entre services et présence d'infection associée aux soins chez les patients.

\section{Aspects éthiques}

Avant le démarrage de la collecte des données, une autorisation de la recherche au CNHU-HKM avait été sollicitée et obtenue. Elle avait été délivrée par le Directeur Général du CNHU-HKM. Les autorités de cet hôpital et le personnel cible avaient été informés de l'intérêt de l'étude; leur consentement avait été obtenu avant l'enquête ; l'anonymat et la confidentialité étaient observés sur toutes les données collectées.

\section{RESULTATS}

Fréquence des infections associées aux soins

La fréquence des infections associées aux soins était de 19 sur 193 soit 9,84\%. L'unité des grands brûlés et le service de l'urologie avaient enregistré plus de cas ; $\left(\mathrm{Chi}^{2}=42,6 ; p=0,001\right)$.

Sur les 19 patients infectés, 11 avaient bénéficié d'une exploration microbiologique; 15 bactéries avaient été identifiées soit un ratio bactérie/patient de 1,36 (Tableau 3).
Site de localisation des infections associées aux soins

Les localisations au niveau des tissus mous et au niveau urinaire étaient les plus fréquentes. Les Staphylococcus aureus et Pseudomonas aeruginosa étaient observés dans les tissus mous et Acinetobacter spp, Escherichia coli et Proteus mirabilis dans le site urinaire.

Par ailleurs, Enterobacter cloacae et Klebsiella pneumoniae étaient souvent identifiés dans le sang (Figure 1).

\section{Sensibilité des bactéries aux antibiotiques}

Des bactéries identifiées, 8 sur les 15 étaient multi résistants aux antibiotiques usuels (sensibles uniquement à l'imipénème de la famille des carbapénèmes) (Tableau 4).

\section{Surveillance des infections associées aux soins}

La surveillance des Infections Associées aux Soins avait atteint un niveau de 2 sur 7 attendu soit $29 \%$; il existait un écart majeur mettant en jeu la qualité de la gestion du risque infectieux associé aux soins (Figure 2).

\section{Qualité de la gestion du risque infectieux associe aux soins}

Le niveau observé de la qualité de gestion du risque infectieux associé aux soins était de 64 sur 100 attendu (64\%) (Figure 3). 


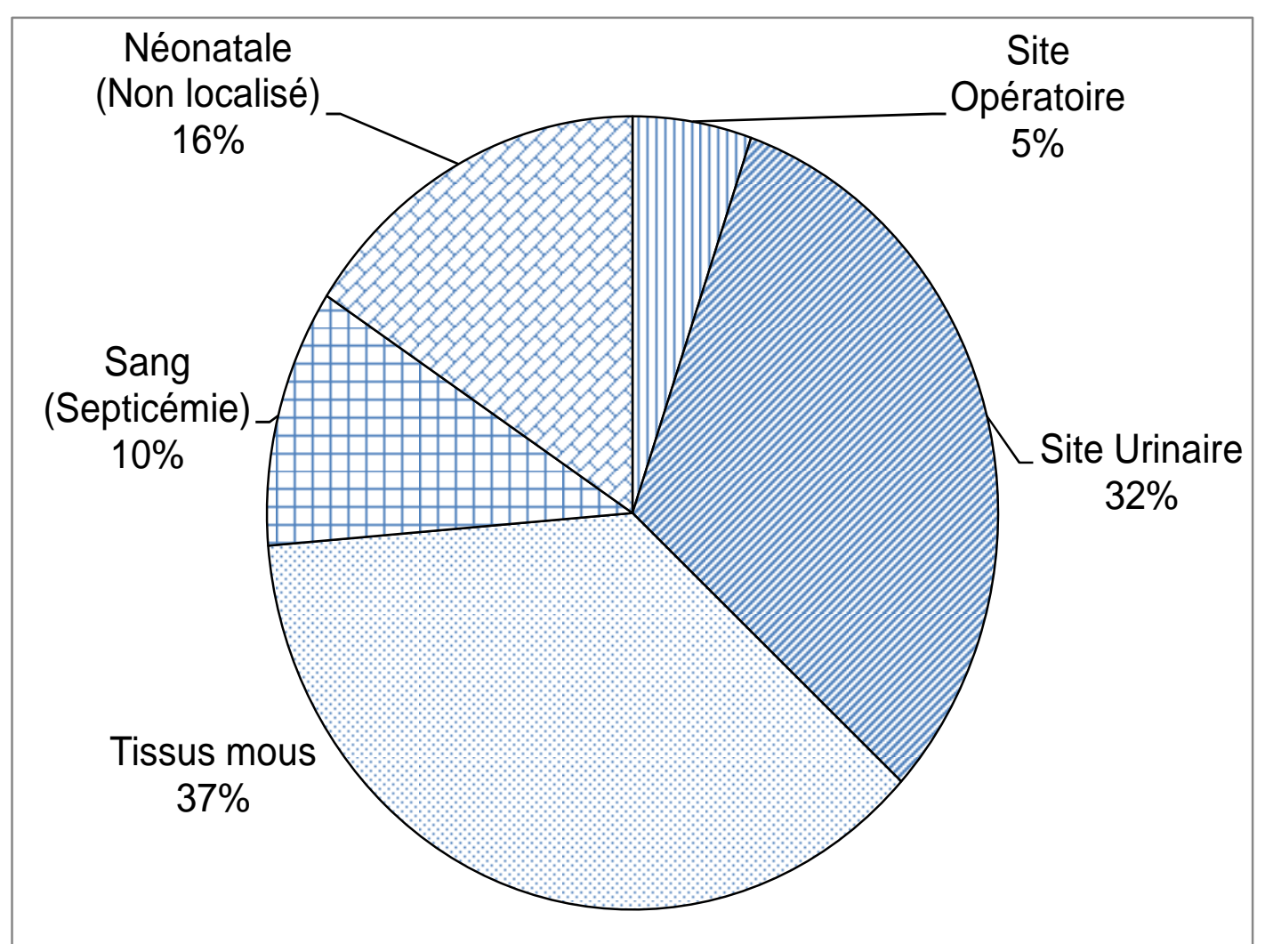

Figure 1 : Répartition par site des cas d'infections associés aux soins.

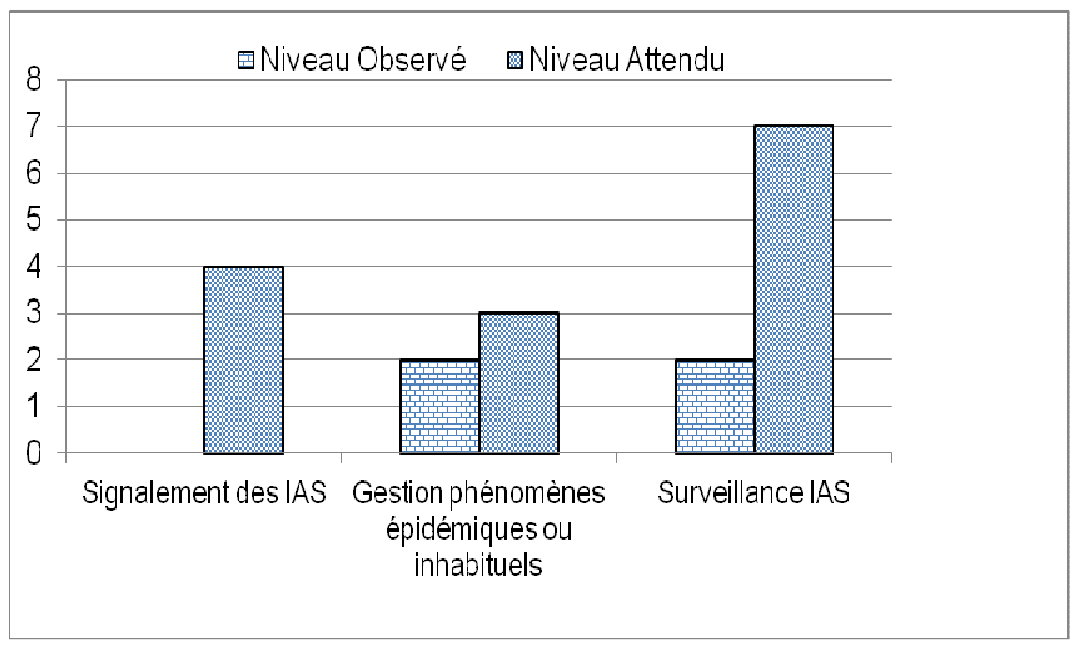

Figure 2 : Surveillance des infections associées aux soins. 


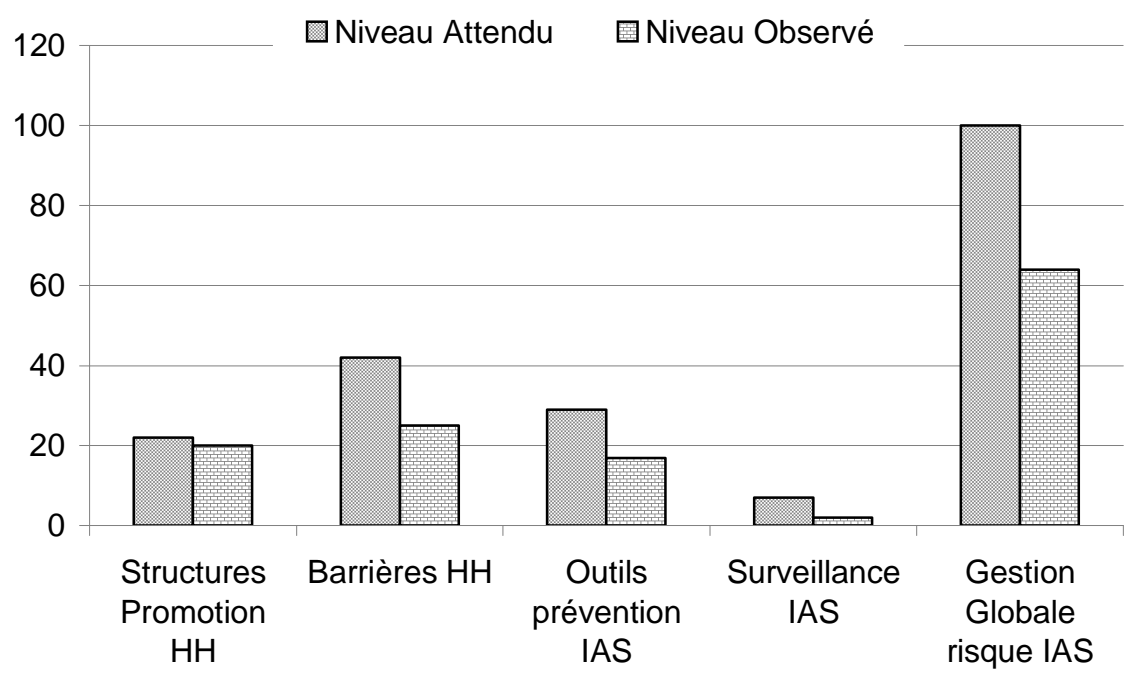

Figure 3 : Qualité de la gestion du risque infectieux associé aux soins. HH : Hygiène hospitalière; IAS : Infections associées aux soins.

Tableau 3 : Répartition des patients infectés en fonction des services.

\begin{tabular}{lccc}
\hline \multirow{2}{*}{ Service } & \multicolumn{2}{c}{ Patients infectés (IAS*) } & \multirow{2}{*}{ Total } \\
\cline { 2 - 3 } & Oui & Non & $64(01,56 \%)$ \\
\hline Urgence & 1 & 63 & $15(53,33 \%)$ \\
Grands brûlés & 8 & 7 & $32(18,75 \%)$ \\
Urologie & 6 & 26 & $42(02,38 \%)$ \\
Viscérale & 1 & 41 & $40(07,5 \%)$ \\
Néonatologie & 3 & 37 & $\mathbf{1 9 3}(\mathbf{0 9 , 8 4 )}$ \\
Total & $\mathbf{1 9}$ & $\mathbf{1 7 4}$ & \\
\hline
\end{tabular}

(*) IAS : Infection associée aux soins.

Tableau 4: Répartition des bactéries identifiées en fonction de la multi résistance aux antibiotiques.

\begin{tabular}{lcc}
\hline Bactéries & $\begin{array}{c}\text { Total des bactéries } \\
\text { identifiées }\end{array}$ & $\begin{array}{c}\text { Nombre de bactéries multi } \\
\text { résistantes }\end{array}$ \\
\hline Klebsiella pneumoniae & 3 & 2 \\
Escherichia coli & 3 & 2 \\
Enterobacter cloacae & 2 & 1 \\
Staphylococcus aureus & 3 & 0 \\
Pseudomonas aeruginosa & 1 & 0 \\
Acinetobacter spp & 2 & 2 \\
Proteus mirabilis & 1 & 1 \\
Total & 15 & 8 \\
\hline
\end{tabular}




\section{DISCUSSION}

Cette étude réalisée sur 193 patients hospitalisés fait une analyse ponctuelle de la situation. Elle présente un intérêt dans le processus de relance des activités de surveillance des infections associées aux soins et service dans cet hôpital. La fréquence des infections associées aux soins et des infectés était respectivement de $10,88 \%$ et $9,84 \%$. Cette fréquence observée était comparable à celle rapportée par les travaux de Biaou (2011) obtenu dans le même hôpital $(6,3 \%$ avec une différence statistiquement significative, $p<0,05)$. La première enquête nationale réalisée par Pittet et al. (2000) rapportait une prévalence de $11,6 \%$ $(p=0,458)$ des infections nosocomiale dans les hôpitaux universitaires suisses. En Afrique, elle varie entre 20 et $60 \%$ et représente la troisième cause de mortalité maternelle, la deuxième cause de mortalité néonatale précoce, et la première cause de morbidité postopératoire (RIPAQS-ICPI, 2011). En 2010, cette prévalence est estimée à 10,9\% au Sénégal, $12 \%$ en Côte d'Ivoire, $10 \%$ au Bénin et $14 \%$ au Mali (RIPAQS-ICPI, 2011). Par contre, la prévalence trouvée dans notre étude était supérieure à celle observée $(5,7 \%)$ par El Rhazi et al. (2007) au CHU Hassan II de Fès au Maroc. Cette fréquence est également supérieure à celles rapportées par les 2 premières enquêtes nationales de prévalence en France (French Prevalence Survey Study Group 2000 et Thiolet et al., 2007). Ces comparaisons doivent néanmoins se faire avec prudence; l'étude de Biaou (2011) était réalisée uniquement en chirurgie viscérale A\&B du même hôpital. Elle portait sur la prévalence des infections du site opératoire. Concernant les études de prévalence réalisées au Maroc et en France; elles avaient été instantanées en un jour suivi de prélèvement des patients hospitalisés et présents le jour de l'enquête avec étude de leurs dossiers cliniques. Ces études avaient été menées dans plusieurs hôpitaux alors que la présente était transversale rétrospective et évaluative portant sur des patients hospitalisés au cours d'un mois.
Localisation et caractéristiques des infections associées aux soins

Les sites de localisation des infections observés étaient les tissus mous (37\%), le site urinaire $(32 \%)$, le sang $(10 \%)$ et les sites opératoires $(5 \%)$. Ces résultats ne concordent pas avec ceux trouvés par plusieurs auteurs. La localisation la plus fréquente des infections associées aux soins se situe au niveau urinaire. Les atteintes de la voie respiratoire suivent, puis la localisation du site opératoire et les autres (Sax et al., 2000).

Toutefois, ces localisations varient selon les services cliniques. Les localisations plus fréquemment constatées en réanimation et classées par ordre décroissant sont le site respiratoire, les infections urinaires, les bactériémies et les infections de site opératoire (Brun-Buisson et al., 2005).

\section{Infections associées aux soins et actes invasifs}

Des 3 cas enregistrés dans l'unité de néonatologie, 2 étaient admis après extraction instrumentale (forceps) à la naissance; aucune infection nosocomiale n'était évoquée dans les 48 heures qui ont suivi leur admission. Guimarães et al. (2011) dans une étude rétrospective à l'hôpital de Sumaré à Sao Paulo avaient identifié une association des infections nosocomiales avec les dispositifs invasifs.

\section{Bactéries}

Les patients ayant subi une infection nosocomiale avaient bénéficié dans une proportion de $57,8 \%$ une exploration microbiologique; Sur les 15 bactéries identifiés, Klebsiella pneumoniae, Escherichia coli et Staphylococcus aureus représentaient 60\%; La projection de ces bactéries ainsi que des sièges d'infection dans le système d'axes factoriels révélait que Enterobacter cloacae et Klebsiella pneumoniae étaient souvent identifiés dans le sang alors que Staphylococcus aureus et Pseudomonas aeruginosa étaient observés dans les tissus mous. Acinetobacter spp, Escherichia coli et Proteus mirabilis avaient surtout été observés au niveau du site urinaire. Les bactéries multi résistantes aux antibiotiques habituels 
représentaient 53\%; Klebsiella pneumoniae, Escherichia coli, Acinetobacter spp, Proteus mirabilis et Enterobacter cloacae étaient les plus dénombrés; Ces résultats se distinguent de ceux obtenus lors de l'enquête nationale de prévalence en 2006 en France (Sax et al., 2000) où Escherichia coli, Staphylococcus aureus et Pseudomonas aeruginosa représentaient $53,5 \%$ alors que la résistance aux antibiotiques était observée par rapport au Staphylococcus aureus, Pseudomonas aeruginosa, les entérobactéries.

Prévention des IAS : Bon usage des antibiotiques

La fréquence de la résistance bactérienne aux antibiotiques est une préoccupation dans cette étude comme pour les hôpitaux en général. Cette situation expose à une large prescription des antibiotiques de dernière génération encore actives; $\mathrm{Ce}$ qui favorise l'émergence de nouvelles résistance. Plusieurs auteurs préconisent le bon usage des antibiotiques pour retarder le phénomène (ANAES, 1996; Schlemmer et al., 2002 ; Joram et al., 2012). En plus de cela, la surveillance et le signalement des cas constituent une étape primordiale à leur prévention (Sax et al., 2000 ; Régnier, 2007 ; Thiolet et al., 2008).

\section{Prévention des infections associées aux soins}

La pratique de l'hygiène des mains par les professionnels soignants est une approche efficace de réduction de ces infections (OMS, 2010). Elle est favorisée par la formation, la disponibilité des ressources favorables à la pratique ainsi que l'information sur les conditions d'utilisation des solutions hydro alcooliques (Pittet et al., 2000 ; Pittet et al., 2001 ; Chevalier et al., 2008). Si l'asepsie est a priori une préoccupation des soignants, tous ne perçoivent pas toutes les facettes du risque d'infection hospitalière.

\section{Conclusion}

$\mathrm{Au}$ terme de cette évaluation, il s'est révélé que le risque d'épidémie de ces infections reste toujours préoccupant avec une fréquence importante de multi résistance des bactéries aux antibiotiques usuels. Il importe la mise en place des mesures efficaces afin de réduire le risque.

\section{REFERENCES}

ANAES. 2003. Manuel d'accréditation des établissements de santé. http://www.hassante.fr/portail/upload/docs/ application, consulté le 03 Avril 2012.

Brun-Buisson et le groupe de travail. 2005. Risques et maîtrise des infections nosocomiales en réanimation. SRLF/SFAR., 14: 463- 471. http://france.elsevier.com/direct/REAUR $\mathrm{G} /$.

Biaou G. 2011. Infections du site opératoire dans les services de chirurgie viscérale $\mathrm{A}$ $\& \mathrm{~B}$ du CNHU-HKM. Thèse de médecine Faculté des Sciences de la Santé Cotonou, Université d'Abomey-Calavi Bénin, Bénin, P .105.

Chevalier B, Margery J, Wade B, Ka S, Diatta B, Gueye M. 2008. Perception du risque nosocomial parmi le personnel hospitalier de l'Hôpital Principal de Dakar. Med. Trop., 68: 593-596.

Dégbey C, Aguèmon B, Ouendo E-M, Makoutodé M, Simon A. 2013. Etude de la qualité du matériel médico-technique utilisé dans les blocs opératoires en vue de la prévention des infections associées aux soins et services au Centre National Hospitalier et Universitaire de Cotonou Benin. Journal de la Société de Biologie Clinique, 018: 29-35.

El Rhazi K, Elfakir S, Berraho M, Tachfouti N, Serhier Z, Kanjaa C. 2007. Prévalence et facteurs de risque des infections nosocomiales au CHU Hassan II de Fès (Maroc). Revue de Santé de la Méditerranée Orientale, 13(1): 56-63.

French prevalence survey Study Group. 2000. Prevalence of nosocomial infections in France: results of the nationwide survey in 1996. J. Hosp. Infect., 46(3): 186-93.

Fontaine D et al. 2001. Améliorer l'hygiène hospitalière. Guide en organisation hospitalière dans les pays en développement. République française, Ministère des affaires étrangères : Paris. 
Guimarães AC, Donalisio MR, Santiago TH, Freire JB. 2011. Mortality associated with nosocomial infection, occurring in a general hospital of Sumaré-SP, Brazil. Rev. Bras. Enferm., 64(5):864-869.

Joram N, de Saint Blanquat L, Stamm D, Launay E, Gras-Le Guen C. 2012.Healthcare-associated infection prevention in pediatric intensive care units: a review Eur. J. Clin. Microbiol. Infect. Dis., 4(3) : 56-63.

Ministère de la santé, de la jeunesse et des sports DGS/DHOS. 2007. Actualisation de la définition des infections nosocomiales. Comité technique des infections nosocomiales et des infections liées aux soins (CTINILS). http://www.sante.gouv.fr/IMG/pdf/rappor tcomplet.pdf.

OMS. 2008. Prévention des infections nosocomiales. Guide pratique. (2 $2^{\text {ème }}$ édn) Genève. http://whqlibdoc.who.int /hq/2008/WHO_CDS_CSR_EPH_2002.1 2_fre.pdf.

OMS. 2010. Résumé des Recommandations de l'OMS pour l'Hygiène des Mains au cours des Soins : Premier Défi Mondial pour la Sécurité des Patients: Un Soin propre est un Soin plus sûr, OMS.

Pittet D, Allegranzi B, Storr J, Donaldson L. 2006. The Global Patient Safety Challenge 2005-2006 "Clean Care is Safer Care". Geneva. International Journal of Infectious Diseases, 10: 419424.

Pittet D, Boyce JM. 2001. Hand hygiene and patient care: Pursuing the Semmelweis legacy. Lancet. Infect. Dis., 1: 9-20.

Pittet D, Hugonnet S. 2000. Effectiveness of a hospital-wide programm to improve compliance with hand hygiene. Lancet, 356: 1307-1312.

Pittet D, Ruef C, Comité de Swiss-NOSO. 2000. Première enquête nationale de prévalence des infections nosocomiales dans les hôpitaux universitaires suisses. Swiss-NOSO, 7(1): 1-5.
Régnier B. 2007. Surveillance des infections nosocomiales en France ; de la prévention à la communication. B.E.H., 14: 12-13.

Réseau d'alerte d'investigation et de surveillance des infections nosocomiales (Raisin).2002-2004. Rapport BMRRaisin http://www.invs.sante.fr/publications/200 6/raisin_2006/index.html

RIPAQS-ICPI. 2011. Rapport final de la conférence internationale sur la prévention et la lutte contre les Infections à Genève. www.ripaqs.net/download/ rapport-final-icpic_2011-sessionripaqs.Pdf.

Roemer MI, Montoya-Aguilar C. 1988. Quality assessment and assurance in primary health care. Geneva WHO Offset Publication, 105: 82-89.

Sax H, Pittet D. 2000. Surveillance des infections nosocomiales. Revue médicale suisse, 702: 1405-1417.

Schlemmer B, Crémieux A, Révillaud O.2002. Promouvoir le bon usage des antibiotiques. A.D.S.P ,38(4) :45-56.

Thiolet JM, Lacavé L, Tronel H, Jarno $\mathrm{P}$, Metzger MH, L'Hériteau F, Gautier C, Coignard B, Raisin ENP. 2007. Enquête nationale de prévalence des infections nosocomiales. Bull Epidemiol Hebd., 5152: 429-32.

Thiolet JM, Poujol I, Bernet C, Cerbenne A, Dumartin C, Raclot I, Sénéchal H, Bouraoui L, Coignard B. 2008. Signalements externes des infections nosocomiales, France, 2006. B.E.H., 265: 30-31.

Vincent A, laprugne-Garcia E. 2008. Infections associées aux soins définition, fréquence et facteurs de risque, Fiches conseils pour la prévention du risque infectieux. CCLIN Sud-Est. , P.5.

Vincent JL. 2003. Nosocomial infections in adult intensive-care units. Lancet, 361: 2068-2077. 